\title{
Effects of 16-bit CT imaging scanning conditions for metal implants on radiotherapy dose distribution
}

\author{
LIUGANG GAO $^{1}$, HONGFEI SUN ${ }^{1}$, XINYE NI $^{1}$, MINGMING FANG ${ }^{2}$ and TAO LIN ${ }^{1}$ \\ ${ }^{1}$ Radiotherapy Department, Second People's Hospital of Changzhou, Nanjing Medical University, Changzhou 213003; \\ ${ }^{2}$ Radiotherapy Department, Changzhou Cancer Hospital of Soochow University, Changzhou 213001, P.R. China
}

Received February 17, 2017; Accepted December 4, 2017

DOI: 10.3892/ol.2017.7586

\begin{abstract}
Dose distribution was calculated and analyzed on the basis of 16-bit computed tomography (CT) images in order to investigate the effect of scanning conditions on CT for metal implants. Stainless steel and titanium rods were inserted into a phantom, and CT images were obtained by scanning the phantom under various scanning conditions: i) Fixed tube current of $230 \mathrm{~mA}$ and tube voltages of 100 , 120 , and $140 \mathrm{kV}$; and ii) fixed tube voltage of $120 \mathrm{kV}$ and tube currents of 180,230 , and $280 \mathrm{~mA}$. The CT value of the metal rod was examined and corrected. In a Varian treatment planning system, a treatment plan was designed on the basis of the CT images obtained under the set scanning conditions. The dose distributions in the phantom were then calculated and compared. The CT value of the metal area slightly changed upon tube current alteration. The dose distribution in the phantom was also similar. The maximum CT values of the stainless steel rod were 14,568, 14,127 and 13,295 HU when the tube voltages were modified to 100,120 , and $140 \mathrm{kV}$, respectively. The corresponding $\mathrm{CT}$ values of the titanium rod were 9,420,8,140 and 7,310 HU. The dose distribution of the radiotherapy plan changed significantly as the tube voltage varied. Compared with the reference dose, the respective maximum dose differences of the stainless steel and titanium rods in the phantom were 5.70 , and $6.62 \%$ when the tube voltage varied. The changes in tube currents resulted in a maximum dose error of $<1 \%$ for stainless steel and titanium. In CT imaging, changes in tube voltages can significantly alter the CT values of metal implants. Thus, this can lead to large errors in radiotherapy dose distributions.
\end{abstract}

Correspondence to: Dr Xinye Ni, Radiotherapy Department, Second People's Hospital of Changzhou, Nanjing Medical University, Changzhou 213003, P.R. China

E-mail: nxy2000@aliyun.com

Key words: scanning condition, metal, 16-bit, dose distribution, CT, radiotherapy

\section{Introduction}

Computed tomography (CT) has been widely used in clinical diagnoses and treatments. In many CT scanners, standard 12-bit depth images have been commonly utilized, and 4,096 $\mathrm{CT}$ values ranging from $-1,024 \mathrm{HU}$ to $3,071 \mathrm{HU}$ have been established. This range can accurately represent different tissues in patients under normal conditions. However, CT values significantly exceed the upper limit of 3,071 HU because of the presence of metal implants in a patient's body. Therefore, different metal implants cannot be distinguished in 12-bit images. In patients with common hip prostheses, implants are characterized by either a high atomic number or high-density metals. The exact CT values of such prostheses cannot also be determined through conventional CT scan; consequently, the calculated dose distributions of radiation are affected $(1,2)$. Therefore, 16-bit CT images are reconstructed by extending the bit depth of CT (3-5), and this reconstruction generates a wide range of $\mathrm{CT}$ values. Coolens (6) also observed that stainless steel, titanium, and other hip prostheses can be differentiated in 16-bit CT images. Metal regions can also be distinguished by adjusting the window width and window level.

In radiotherapy, dose distribution is calculated on the basis of a patient's CT image (7) in a treatment planning system (TPS). In the TPS, a CT image is converted to the corresponding electron density on the basis of a calibrated CT with a relative electron density (CT-ED) curve, which is obtained under a certain standard scanning condition. Patients are usually examined under different scanning conditions depending on clinical requirements. The frequent upgrades of hardware and software for CT scanners and TPS may require recalibration procedures, which are hard to satisfy. In practice, a conversion table is usually fixed and a scanning condition similar to a standard condition is selected whenever possible. Nevertheless, this procedure may cause errors in dose delivery. Varying CT scanning conditions may alter the CT value of the same material (8). For instance, Zurl (9) demonstrated that a change in tube voltage results in variations of up to $20 \%$ in $\mathrm{HU}$ and thus yields a maximum dose error of $1.5 \%$. However, dose errors may increase remarkably in patients with high-density metal implants, and such errors are detrimental to patients undergoing radiotherapy. In the present study, stainless steel and titanium rods, which are commonly used implanted materials, were scanned and analyzed under various CT scanning 
conditions. Differences in dose distributions for CT images were further analyzed.

\section{Materials and methods}

Experimental materials. We used a computerized imaging reference system (CIRS) intensity-modulated radiation therapy-verified phantom (Fig. 1) produced by CIRS, Inc., USA. A cylindrical metal rod, a stainless steel rod, or a titanium alloy rod was inserted into the center of the phantom. A high-density rod with a bone-like tissue and two cylindrical rods composed of a material similar to that of the phantom were also utilized. The phantom was scanned with SOMATOM Definition Flash CT (SIEMENS). The extended bit depth function was chosen, and a 16-bit CT image was reconstructed. The axial scanning mode was applied. The collimator width was $64.0 \times 0.6 \mathrm{~mm}$ and the X-ray tube rotation time was $0.5 \mathrm{~s}$ /rotation. The scanning layer thickness was $2 \mathrm{~mm}$, and the image reconstruction matrix was $512 \times 512$.

Experimental method. After the metal rod was inserted into the phantom, the tube voltages or currents were altered and the other scanning conditions were unchanged. The CT images were obtained under different conditions during the experiment. The tube current was fixed at $230 \mathrm{~mA}$, and the tube voltages were 100,120 , and $140 \mathrm{kV}$. The tube voltage was fixed at $120 \mathrm{kV}$, and the tube currents were set to 180,230 , and $280 \mathrm{~mA}$. CT image data in DICOM format were inputted into MATLAB 8.3. The relationship between the CT value distribution of the metal parts and the scanning conditions was analyzed. The standard scanning conditions were set as $120 \mathrm{kV}$ and $230 \mathrm{~mA}$, and the absolute and relative differences in $\mathrm{CT}$ values were calculated when the tube voltage and tube current were changed.

$$
\begin{gathered}
d=x-x_{120 \mathrm{kV}, 230 \mathrm{~mA}} \\
r=\frac{d}{x_{120 \mathrm{KV}, 230 \mathrm{~mA}}} \times 100 \% .
\end{gathered}
$$

where $\mathrm{x}$ is the CT value of the material under each scanning condition, $\mathrm{x}_{120 \mathrm{kV}, 230 \mathrm{~mA}}$ is the CT value of the material under the standard scanning conditions $120 \mathrm{kV}$ and $230 \mathrm{~mA}, \mathrm{~d}$ is the absolute difference between these values, and $\mathrm{r}$ is the relative difference between these values. Throughout this text, the CT values are the theoretical Hounsfield number (HU) plus $1,000(6,10)$; i.e., air $=0$ and water $=1,000$. The $\mathrm{CT}$ value of the phantom is similar to water.

The densities of the titanium alloy and stainless steel rods were high, and their corresponding CT values were significantly higher than that of normal tissues. The metal parts could be segmented on the basis of $4000 \mathrm{HU}$ threshold. In the phantom, the CT value of the metal parts fluctuated greatly because of the serious 'cupping artifact' and inaccuracy. For the cylindrical metal rod, the maximum CT value at the edge of the metal region is close to the actual value (5). To correct the 'cupping artifact', we uniformly set the CT values of the segmented metal area to the maximum CT value of the metal region. The corrected CT images were then inputted into the Varian treatment planning system (Varian Eclipse edition 11.0). In the TPS, the CT-ED curve (Fig. 2) calibrated at $120 \mathrm{kV}$ and $230 \mathrm{~mA}$ was used in

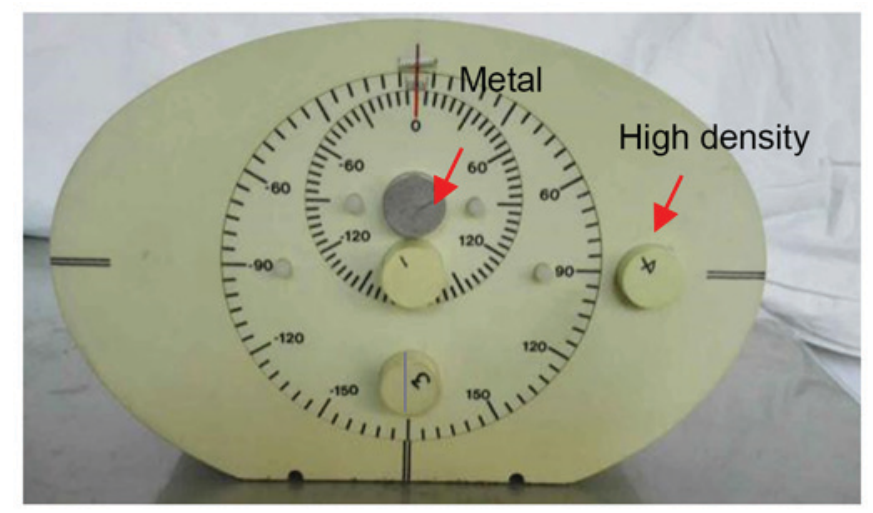

Figure 1. Phantom used in the experiment.

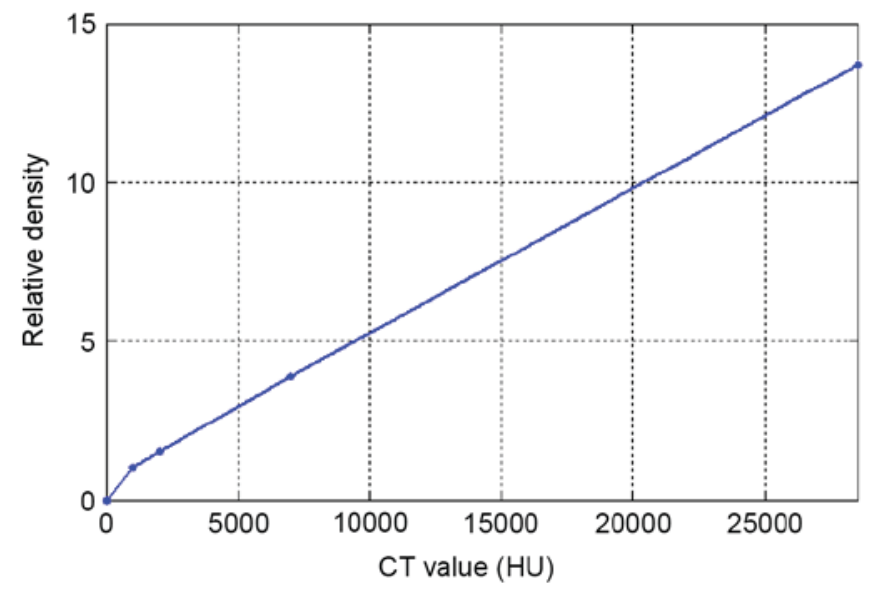

Figure 2. CT to relative electron density curve derived from a 16-bit CT image.

dose calculation. A radiotherapy treatment plan was designed on the basis of these images. A $0^{\circ}$ single irradiation field was used, the source to skin distance (SSD) was $100 \mathrm{~cm}$, and the machine output quality was $200 \mathrm{MU}$. The radiation field was $10 \mathrm{x} 10 \mathrm{~cm}$, and the X-ray energy was $6 \mathrm{MV}$. Dose distribution was calculated using an anisotropic analytic algorithm.

\section{Results}

CT value distribution in the phantom. Fig. 3 depicts the CT value distribution in the phantom with a stainless steel rod under different scanning conditions. The serious 'cupping artifact' was observed in uncorrected CT images Fig. 3A and C. The tube voltage ranged from $100-140 \mathrm{kV}$, and the CT values of the metals varied significantly. On the edge of the metal area, the largest CT value was detected at $100 \mathrm{kV}$ and the smallest CT value was recorded at $140 \mathrm{kV}$. By contrast, the $\mathrm{CT}$ value of the metal center was decreased at $100 \mathrm{kV}$ and increased at $140 \mathrm{kV}$. The changes in the tube current resulted in slight deviations in the metal region.

A region of interest ( $\mathrm{ROI}$ ) in the phantom, as shown in the rectangular red region far from the metal in Fig. 3A, was analyzed. The mean CT values of the ROI were 999, 998, and $997 \mathrm{HU}$ when the tube voltages were 100, 120, and $140 \mathrm{kV}$, respectively. The changes in the tube voltage resulted 
Table I. Maximum CT values of stainless steel and titanium under various scanning conditions. Differences calculated through Eqs. (1) and (2).

\begin{tabular}{lccccccr}
\hline & \multicolumn{3}{c}{ Stainless steel } & & \multicolumn{3}{c}{ Titanium } \\
\cline { 2 - 3 } Scanning conditions & CT value & $\mathrm{d}(\mathrm{HU})$ & $\mathrm{r}(\%)$ & & CT value & $\mathrm{d}(\mathrm{HU})$ & $\mathrm{r}(\%)$ \\
\hline $120 \mathrm{kV}, 230 \mathrm{~mA}$ & 14,127 & - & - & 8,140 & - & - \\
$100 \mathrm{kV}, 230 \mathrm{~mA}$ & 14,568 & 441 & 3.12 & & 9,420 & 1,280 & 15.72 \\
$140 \mathrm{kV}, 230 \mathrm{~mA}$ & 13,295 & -832 & -5.89 & & 7,310 & -830 & -10.20 \\
$120 \mathrm{kV}, 180 \mathrm{~mA}$ & 14,107 & -20 & -0.14 & & 8,080 & -60 & -0.74 \\
$120 \mathrm{kV}, 280 \mathrm{~mA}$ & 14,183 & 56 & 0.40 & & 8,140 & 0 & 0 \\
\hline
\end{tabular}
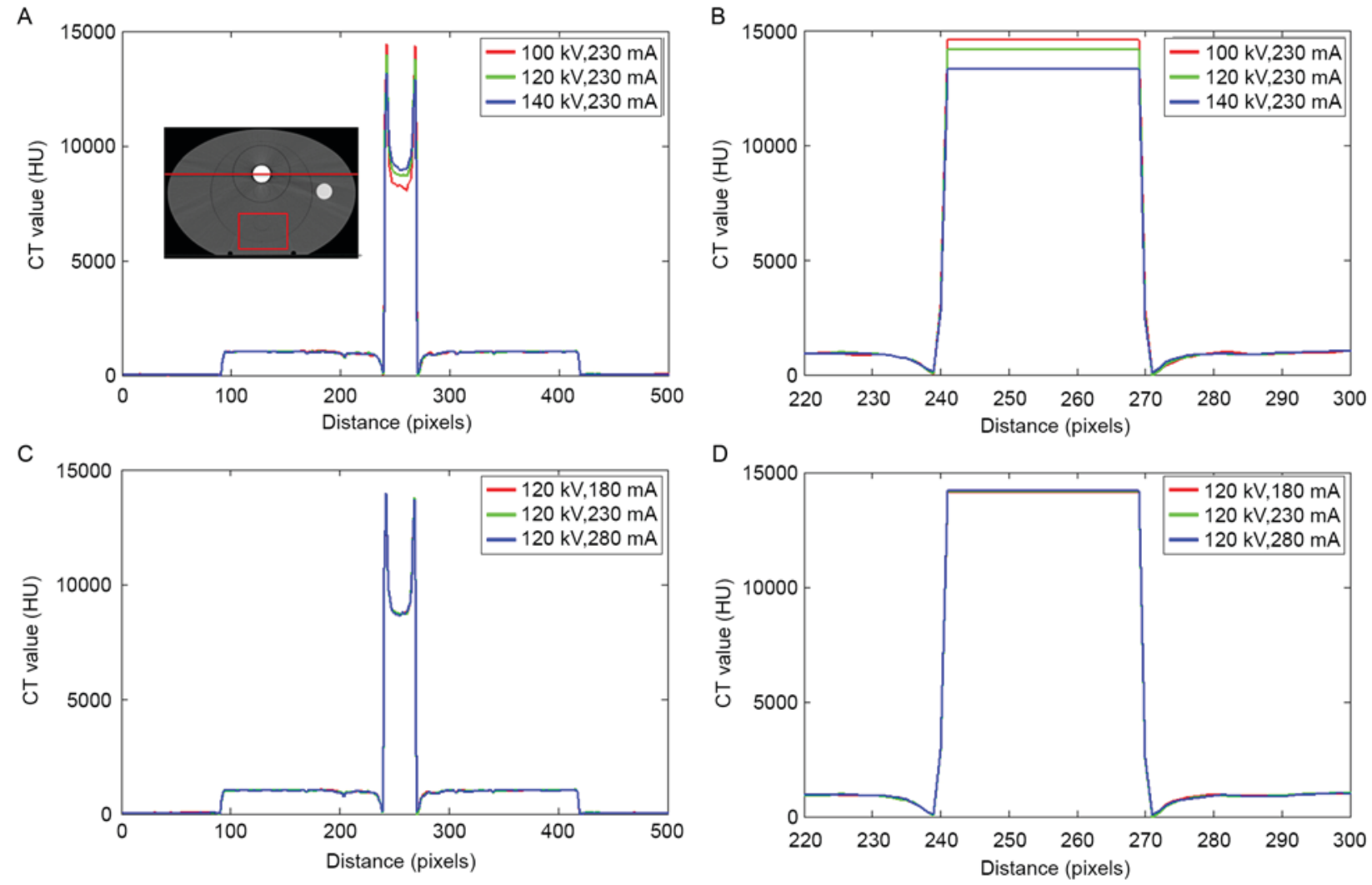

Figure 3. Distribution of CT values for the phantom with a stainless steel rod. (A) With fixed tube current and different tube voltages. (B) The CT values of a metal area are corrected in (A). (C) With fixed tube voltage and different tube currents. (D) The CT values of a metal area are corrected in (C).

in a slight variation of the CT values at low densities. These findings are consistent with those in a previous study (9). For the bone equivalent insert, the mean CT values were 1,811 , 1,741 , and $1,700 \mathrm{HU}$ when the tube voltages were 100,120 , and $140 \mathrm{kV}$, respectively. The changes in the tube voltage resulted in larger deviations as density increased.

The CT value in the phantom with a titanium rod is illustrated in Fig. 4. Similar to those shown in Fig. 3, large deviations were observed in the titanium rod as the tube voltage changed. The CT value of the metal was slightly influenced by the tube current.

The differences in the CT values of stainless steel and titanium under various scanning conditions are shown in Table I. The CT value of the metals was corrected with the maximum $\mathrm{CT}$ values in the metal region. With different tube voltages, the deviations in HU reached 5.89 and $15.72 \%$ for stainless steel and titanium, respectively. Variations smaller than $1 \%$ in $\mathrm{HU}$ were caused by changes in the tube currents for both stainless steel and titanium.

Dose distribution in the phantom. In the TPS, the CT image was converted to the corresponding electron density via the CT-ED curve (11-13) calibrated at $120 \mathrm{kV}$ and $230 \mathrm{~mA}$, and dose distribution was calculated.

The calculated dose distributions in the phantom with stainless steel rod under different scanning conditions are shown in Fig. 5 (top row). The bottom row in Fig. 5 reveals the dose difference under various scanning conditions compared with the standard conditions of $120 \mathrm{kV}$ and $230 \mathrm{~mA}$. The dose distribution for titanium rod is displayed in Fig. 6. As the tube 

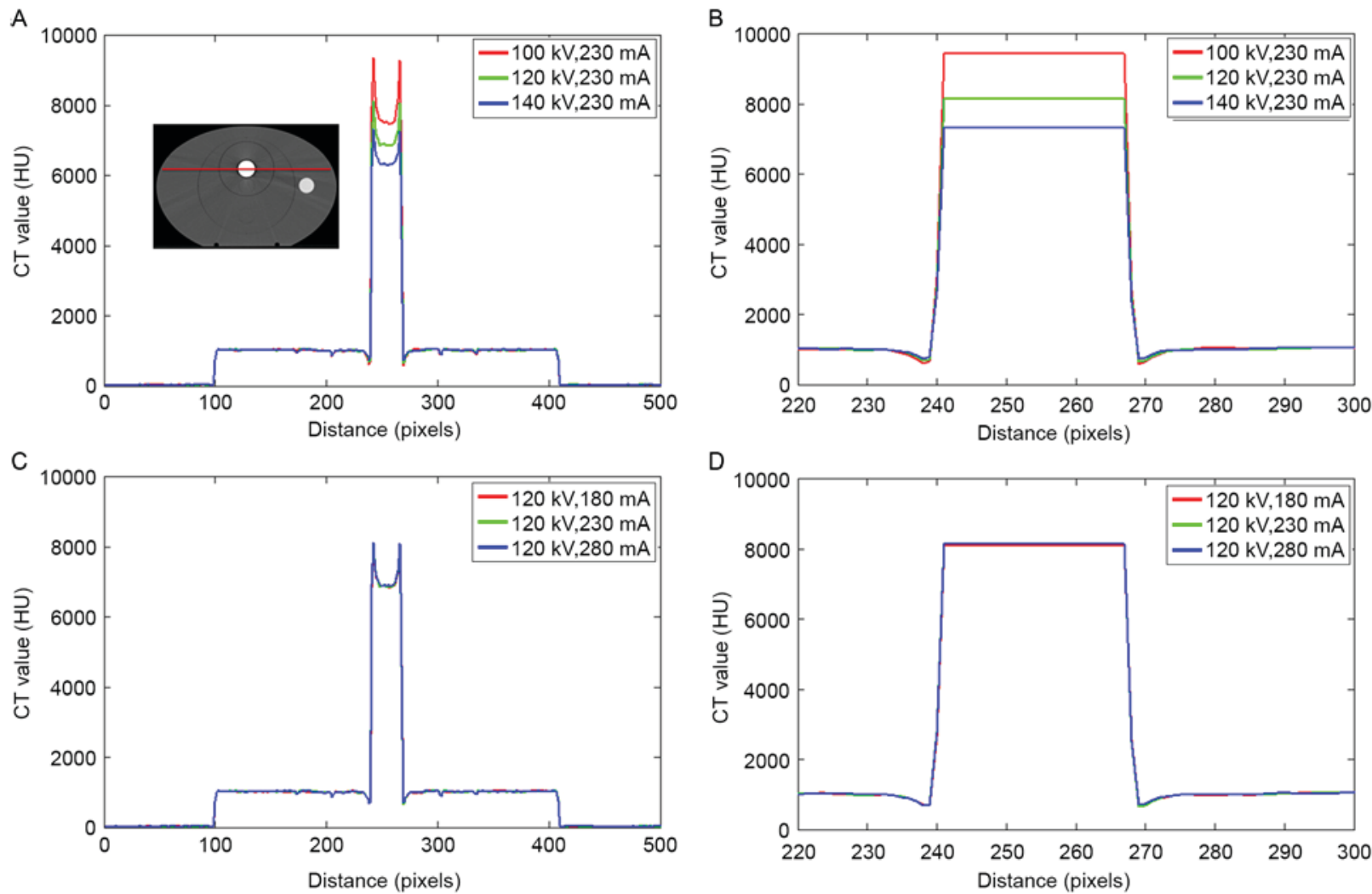

Figure 4. Distribution of CT values for the phantom with a titanium rod. (A) With fixed tube current and different tube voltages. (B) The CT values of a metal area are corrected in (A). (C) With fixed tube voltage and different tube currents. (D) The CT values of a metal area are corrected in (C).

Dose distribution

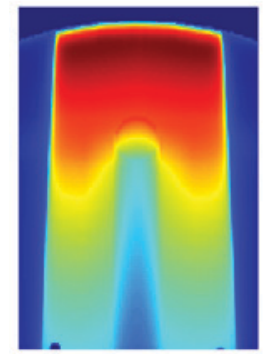

$120 \mathrm{kV}, 230 \mathrm{~mA}$

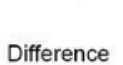

Difference

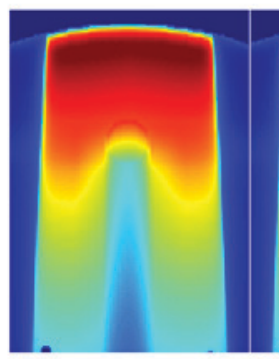

$100 \mathrm{kV}, 230 \mathrm{~mA}$

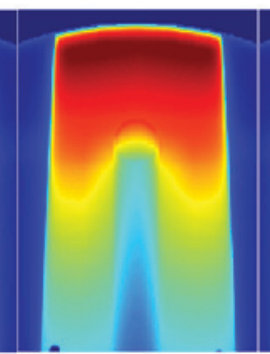

$120 \mathrm{kV}, 180 \mathrm{~mA}$

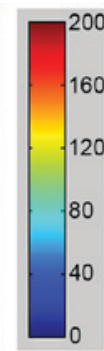

$120 \mathrm{kV}, 280 \mathrm{~mA}$

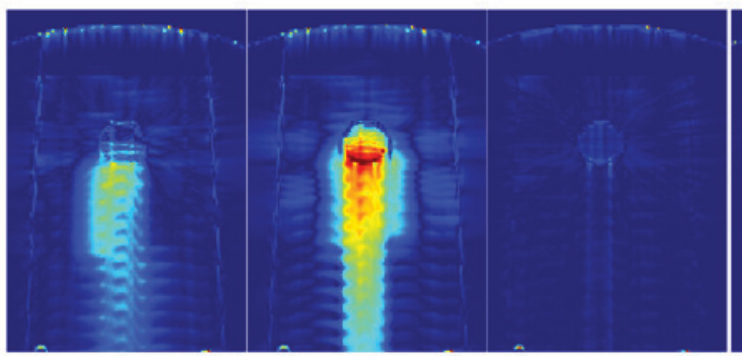

$100 \mathrm{kV}, 230 \mathrm{~mA}$

$140 \mathrm{kV}, 230 \mathrm{~mA}$

$120 \mathrm{kV}, 180 \mathrm{~mA}$

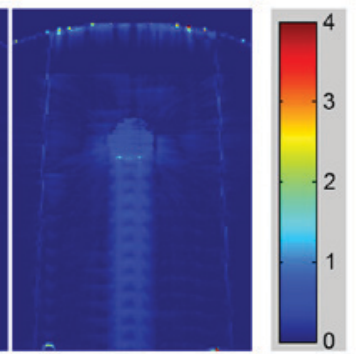

$120 \mathrm{kV}, 280 \mathrm{~mA}$

Figure 5. Calculated dose distributions (top row) in the phantom with a stainless steel rod under various scanning conditions. Differences in dose distribution (bottom row) between $120 \mathrm{kV}$ and $230 \mathrm{~mA}$ and other scanning conditions. Legends indicate dose in cGy.

voltage changed, the dose errors resulting from the deviations of CT values were observed in stainless steel and titanium. The major error was detected in the region downstream of the metal rod. Small errors caused by the tube current were also detected.
Fig. 7 summarizes the dose profiles following the beam trajectory in the phantom with stainless steel under different scanning conditions. The profiles were normalized to the maximum dose before they were compared. A peak was 
Dose distribution

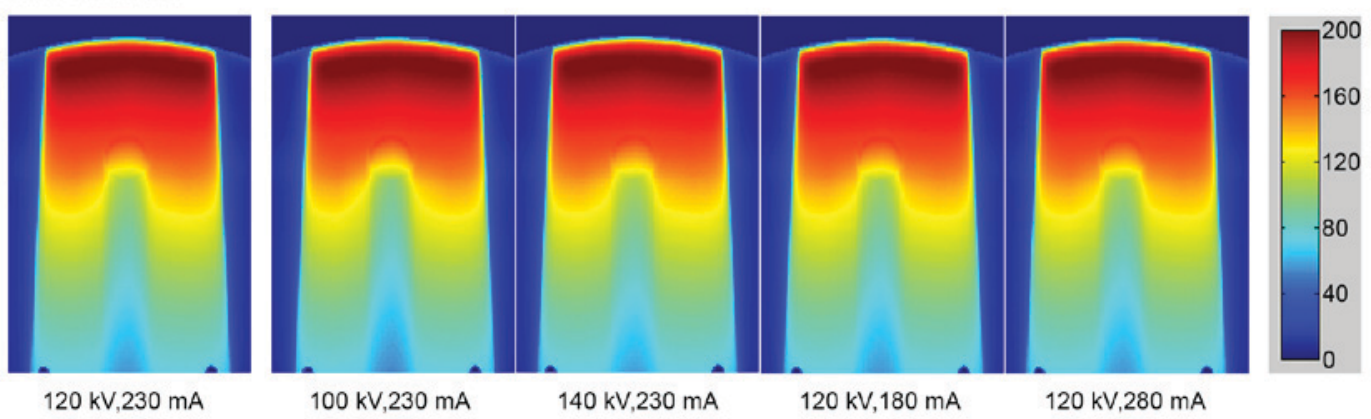

Difference

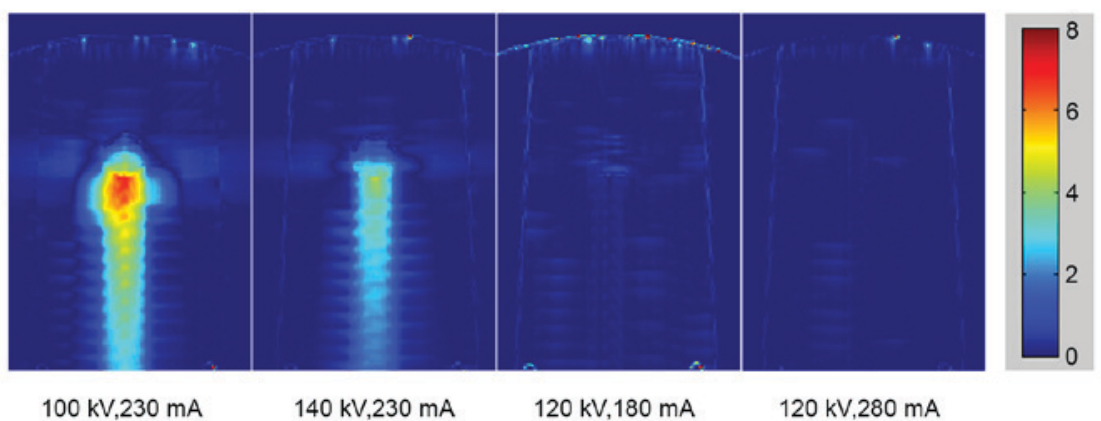

Figure 6. Calculated dose distributions (top row) in the phantom with a titanium rod under various scanning conditions. Differences in dose distributions (bottom row) between the $120 \mathrm{kV}$ and $230 \mathrm{~mA}$ and other scanning conditions. Legends indicate dose in cGy.

A

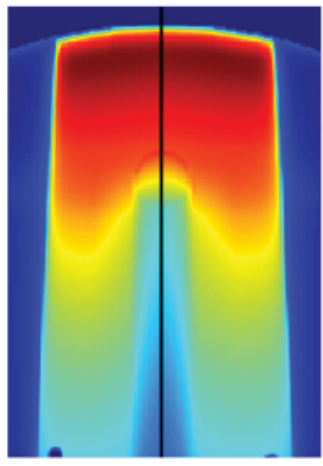

C

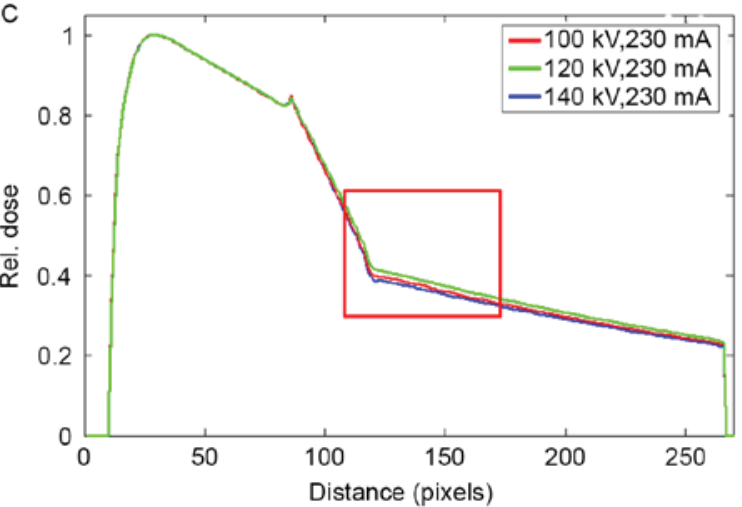

B

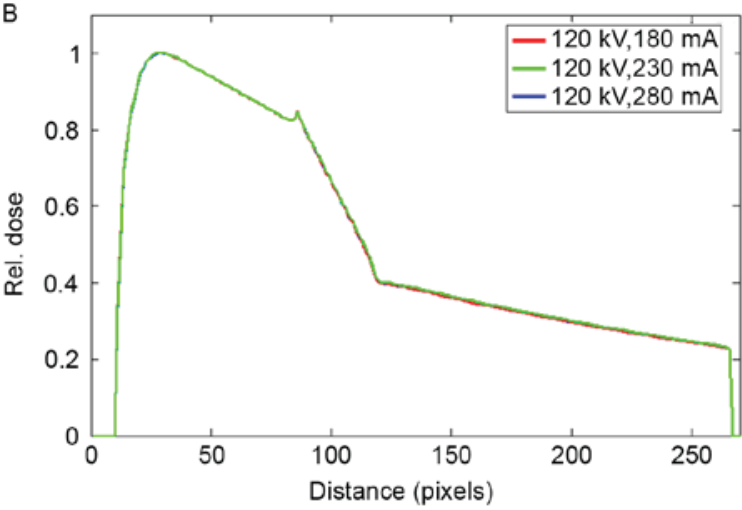

D

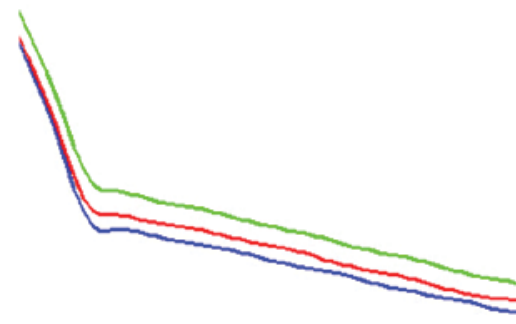

Figure 7. Dose profile in the phantom with a stainless steel rod. (A) Region of interest. (B) With fixed tube voltage and different tube currents. (C) With fixed tube current and different tube voltages. (D) Magnification of the region in (C).

observed at the tissue-metal interface. At different tube currents, the curves of the dose profiles almost completely overlapped. As the tube currents changed to 180 and $280 \mathrm{~mA}$, the respective maximum dose errors were 0.55 and $0.98 \%$. The changes in tube voltages resulted in visible differences in the profiles. The maximum dose errors of 2.85 and $5.70 \%$ 


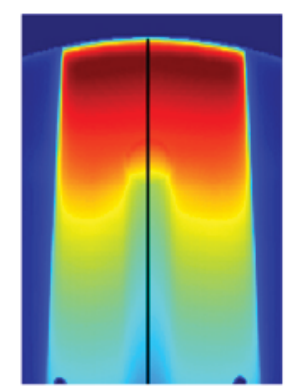

b

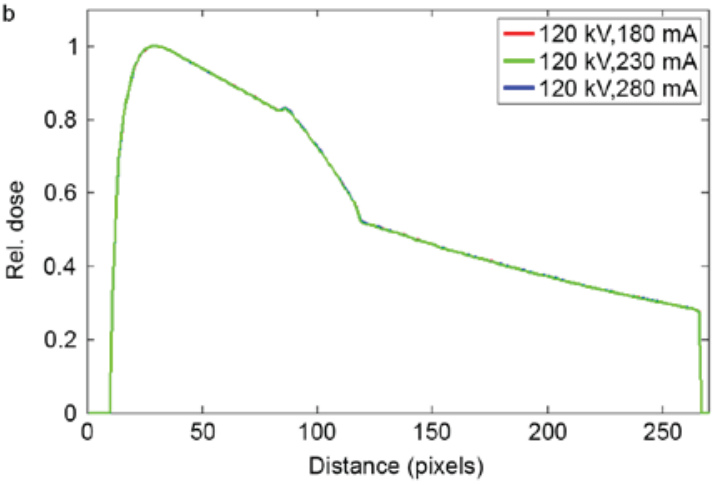

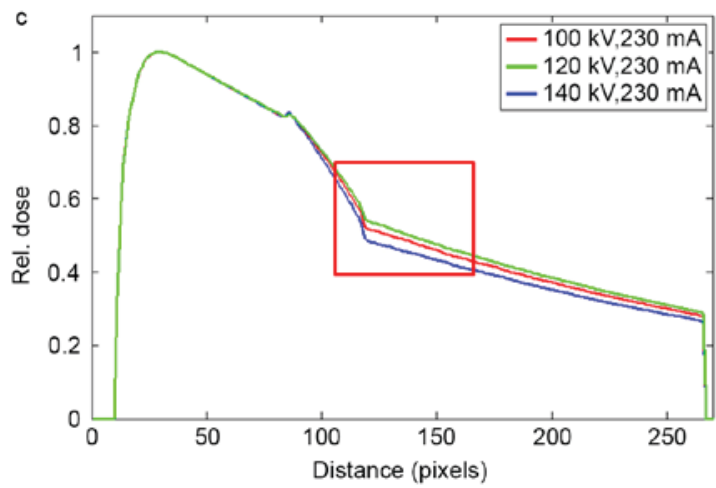

d

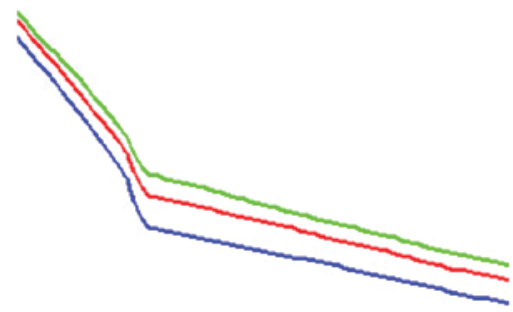

Figure 8. Dose profile in the phantom with a titanium rod. (A) Region of interest. (B) With fixed tube voltage and different tube currents. (C) With fixed tube current and different tube voltages. (D) Magnification of the region in (C).

were observed at 100 and $140 \mathrm{kV}$, respectively. The mean dose errors in the downstream of the metal rod were 1.99 and $4.72 \%$ at 100 and $140 \mathrm{kV}$, respectively.

The dose profile in the phantom with titanium rod is displayed in Fig. 8. Similar to Fig. 7, the dose profiles almost overlapped as the tube current changed, and significant differences in the profiles were attributed to the variations in tube voltages. The maximum dose errors of 0.61 and $0.09 \%$ were observed at 180 and $280 \mathrm{~mA}$, respectively. The CT values of titanium did not vary as the tube current was changed to $280 \mathrm{~mA}$ (Table I). A dose error of up to $0.09 \%$ was a random error, not a result of the changes in tube current. The maximum dose errors of 6.62 and $4.37 \%$ were observed at 100 and $140 \mathrm{kV}$, respectively. The mean dose errors in the downstream of the metal rod were 5.26 and $3.31 \%$ at 100 and $140 \mathrm{kV}$, respectively.

\section{Discussion}

The dependence of $\mathrm{CT}$ values on scanning conditions has been extensively investigated in normal tissues $(14,15)$. Dose errors caused by different scanner settings should be considered in TPS (16). For metal implants, variations in CT values as a result of different scanning conditions are larger than those in normal tissues. Consequently, dose errors are increased.

The present study aimed to evaluate the effects of different scanning conditions on CT values and dose errors. For stainless steel and titanium rods, CT values were slightly influenced by tube currents. Thus, a maximum dose error of less than $1 \%$ was obtained. However, the influence of the changes in tube voltage on CT values should also be determined. In this study, the CT values of the metal decreased as the tube voltage increased. As the tube voltage was changed, the energy of the X-ray photon emitted by a CT machine was altered. Attenuation coefficients varied with X-ray energies. As a result, CT values varied. For high-density materials, a decrease in tube voltage causes a reduction of X-ray photon energy, and the attenuation coefficient of a material is increased as CT values increase. For commonly used implant materials, such as stainless steel and titanium, changes in tube voltages affected CT values. Thus, evident dose errors were obtained. The changes in the tube voltage yielded maximum dose errors of 5.70 and $6.62 \%$ for stainless steel and titanium, respectively. These findings were much larger than those in a previous study (9), which showed a maximum dose error of $1.5 \%$ in normal tissues.

Metal CT values can be determined through 16-bit CT imaging. This method can also be applied to distinguish stainless steel, titanium, and other metal implants with different densities. The $\mathrm{CT}$ value of a metal implant changes significantly and the dose distributions based on CT images considerably differ when tube voltage is altered during CT imaging. The CT value of a metal changes slightly when tube current is altered to a certain extent. Thus, dose distributions slightly vary. In the radiotherapy of patients with metal implants, CT scanning should be executed under a fixed tube voltage to ensure accuracy of the calculated dose. The tube voltage must be same as the condition under which the CT-ED relationship was calibrated.

\section{Acknowledgements}

The present study was supported by the Natural Science Foundation of Jiangsu Province Research of China (grant no. BK20151181), High-Level Medical Talents Training. 
Project of Changzhou (grant no: 2016CZLJ004) and the Municipal Social Development Project of the Changzhou City, Jiangsu Province, China (grant no. CJ20160029).

\section{References}

1. Keall PJ, Chock LB, Jeraj R, Siebers JV and Mohan R: Image reconstruction and the effect on dose calculation for hip prostheses. Med Dosim 28: 113-117, 2003.

2. Keall PJ, Siebers JV, Jeraj R and Mohan R: Radiotherapy dose calculations in the presence of hip prostheses. Med Dosim 28 107-112, 2002.

3. Glide-Hurst C, Chen D, Zhong $\mathrm{H}$ and Chetty IJ: Changes Realized from extended bit-depth and metal artifact reduction in CT. Med Phys 40: 061711, 2013.

4. Link TM, Berning W, Scherf S, Joosten U, Joist A, Engelke K and Daldrup-Link HE: CT of metal implants: Reduction of artifacts using an extended CT scale technique. J Comput Assist Tomogr 24: 165-172, 2000.

5. Paudel MR, Mackenzie M, Fallone BG and Rathee S: Evaluation of normalized metal artifact reduction (NMAR) in kVCT Using MVCT prior images for radiotherapy treatment planning. Med Phys 40: 081701, 2013.

6. Coolens C and Childs PJ: Calibration of CT Hounsfield units for radiotherapy treatment planning of patients with metallic hip prostheses: The use of the extended CT-scale. Phys Med Biol 48: 1591-1603, 2003.

7. Schneider U, Pedroni E and Lomax A: The calibration of CT hounsfield units for radiotherapy treatment planning. Phys Med Biol 41: 111-124, 1996.

8. Thomas SJ: Relative electron density calibration of CT scanners for radiotherapy treatment planning. Br J Radiol 72: 781-786, 1999.

9. Zurl B, Tiefling R, Winkler P, Kindl P and Kapp KS: Hounsfield units variations: Impact on CT-density based conversion tables and their effects on dose distribution. Strahlenther Onkol 190 88-93, 2014.
10. Newhauser WD, Giebeler A, Langen KM, Mirkovic D and Mohan R: Can megavoltage computed tomography reduce proton range uncertainties in treatment plans for patients with large metal implants? Phys Med Biol 53: 2327-2344, 2008.

11. Ebert MA, Lambert J and Greer PB: CT-ED conversion on a GE Lightspeed-RT scanner: Influence of scanner settings. Australas Phys Eng Sci Med 31: 154-159, 2008.

12. Saw CB, Loper A, Komanduri K, Combine T, Huq S and Scicutella C: Determination of CT-to-density conversion relationship for image-based treatment planning systems. Med Dosim 30: 145-148, 2005.

13. Constantinou C, Harrington JC and DeWerd LA: An electron density calibration phantom for CT-based treatment planning computers. Med Phys 19: 325-327, 1992.

14. Skrzyński W, Zielińska-Dabrowska S, Wachowicz M, Slusarczyk-Kacprzyk W, Kukołowicz PF and Bulski W: Computed tomography as a source of electron density information for radiation treatment planning. Strahlenther Onkol 186: 327-333, 2010.

15. Zabel-du Bois A, Ackermann B, Hauswald H, Schramm O, Sroka-Perez G, Huber P, Debus J and Milker-Zabel S: Influence of intravenous contrast agent on dose calculation in 3-D treatment planning for radiosurgery of cerebral arteriovenous malformations. Strahlenther Onkol 185: 318-324, 2009.

16. Ramm U, Damrau M, Mose S, Manegold KH, Rahl CG and Böttcher HD: Influence of CT contrast agents on dose calculations in a 3D treatment planning system. Phys Med Biol 46: 2631-2635, 2001

(i)(9) This work is licensed under a Creative Commons Attribution-NonCommercial-NoDerivatives 4.0 International (CC BY-NC-ND 4.0) License. 